\title{
PROJETO AGROAÇÃO: INCENTIVANDO A ALIMENTAÇÃO SAUDÁVEL E A CONSERVAÇÃO DA BIODIVERSIDADE EM CURITIBANOS/SC
}

\author{
Karine Louise dos Santos \\ Universidade Federal de Santa Catarina \\ karine.santos@ufsc.br \\ Sindi Elen Senff \\ Universidade Federal de Santa Catarina \\ sindielen@hotmail.com
}

\author{
Enio Paulo Belotto \\ Universidade Federal de Santa Catarina \\ enio.belotto@ufsc.br \\ Danielle Remor \\ Universidade Federal de Santa Catarina \\ danielle_remor@hotmail.com
}

\begin{abstract}
Resumo
A discussão sobre segurança alimentar e educação ambiental vêm crescendo constantemente, devido a baixa diversidade de frutas, hortaliças e cereais na dieta alimentar, devido a problemas de saúde, e aos problemas ambientais vinculados ao sistema produtivo. Por isso, existe a necessidade de incentivar projetos de educação que discutam a alimentação saudável e a possibilidade de obtenção de alimentos de qualidade, em consonância com a conservação dos recursos naturais e da biodiversidade. Com esse objetivo, as atividades do projeto AgroAção estão sendo conduzidas na horta agroecológica e na Trilha do Pessegueirinho localizadas na Universidade Federal de Santa Catarina, Campus de Curitibanos, juntamente com crianças entre 7 a 12 anos. Até o momento, 442 alunos participaram das atividades do projeto em 13 visitas, beneficiando cinco escolas públicas do município de Curitibanos/SC. Essa troca de experiência está proporcionando aprendizado para os alunos e professores no que se refere a produção de alimentos e a conservação dos recursos naturais e biodiversidade. Palavras-chave: Horta. Alimentação. Conservação. Sustentável.
\end{abstract}

\section{AGROATION PROJECT: ENCOURAGING HEALTHY FEEDING AND BIODIVERSITY CONSERVATION IN CURITIBANOS/SC}

\begin{abstract}
The discussion about food security and environmental education has been growing constantly, due to the low diversity of fruits, vegetables and cereals our diet, health problems, and environmental problems linked to the production system. Therefore, there is a need to encourage education projects that discuss healthy and the possibility of obtaining quality food in consonance with the conservation of natural resources and biodiversity. With this goal in mind, the activities of the AgroAção project are being conducted in the agroecological garden and in the Pessegueirinho Trail located at UFSC, Curitibanos Campus, together with children with age between 7 and 12 years old. Until the moment, 442 students participated in project activities in 13 visits, benefiting five municipal public schools of Curitibanos / SC. This exchange of experience is providing learning to students and teachers as it relates to food production and conservation of natural resources and biodiversity.
\end{abstract}

Keywords: Garden. Feeding. Conservation. Sustainable.

\section{PROYECTO AGROACIÓN: INCENTIVANDO LA ALIMENTACIÓN SALUDABLE Y LA CONSERVACIÓN DE LA BIODIVERSIDAD EN CURITIBANOS/SC}

\footnotetext{
Resumen

La discusión sobre seguridad alimentaria y educación ambiental viene creciendo constantemente, debido a la baja diversidad de frutas, hortalizas y cereales la dieta alimentaria, los problemas de salud, ya los problemas ambientales vinculados al sistema productivo. Por eso, existe la necesidad de incentivar proyectos de educación que discutan la alimentación sana y la posibilidad de obtener alimentos de calidad, en consonancia con la conservación de los recursos naturales y de la biodiversidad. Con este objetivo en mente, las actividades del proyecto AgroAção se están llevando a cabo en la huerta agroecológica y en el Camino del Pessegueirinho ubicadas en la UFSC, Campus de Curitibanos, con niños de entre 7 y 12 años. Hasta el momento, 442 alumnos participaron en las actividades del proyecto en 13 visitas, beneficiando cinco escuelas públicas del municipio de Curitibanos / SC. Este intercambio de experiencia está proporcionando aprendizaje para los alumnos y profesores en lo que se refiere a la producción de alimentos y la conservación de los recursos naturales y la biodiversidad.

Palavras clave: Horta. Alimentación. Conservación. Sostenible.
} 
Projeto Agroação: incentivando a alimentação saudável e a conservação da biodiversidade em Curitibanos/SC

\section{INTRODUÇÃO}

É importante conscientizar a todos, principalmente as crianças, sobre as dificuldades enfrentadas para garantir uma alimentação saudável, na qual se destacam a condição financeira, a falta de conhecimento, o estreitamento da base alimentar e o distanciamento dos consumidores do sistema produtivo. Todos esses aspectos refletem, por exemplo, em um baixo consumo de frutas e verduras, o qual se encontra inferior ao recomendado em 2003 pela OMS (Organização Mundial da Saúde) e a FAO (Organização das Nações Unidas para Alimentação e Agricultura), de 400 gramas por pessoa diariamente (BENTO; ESTEVES ; FRANÇA, 2015; CARVALHO FILHO, 1995;MARANHÃO et al., 2014)

Além disso, há também as dificuldades decorrentes das mudanças climáticas, onde a agricultura enfrenta sérios problemas em garantir produção de qualidade. E para que este cenário mude é necessário mudanças positivas no manejo agrícola que proporcione uma maior conservação dos recursos naturais e da biodiversidade, garantindo a produtividade agrícola, a segurança alimentar e, minimizando os problemas ambientais (ALTIERI; KOOHAFKAN, 2008; LIMA, 2002; PINTO; ASSAD, 2009).

Nesse contexto, a horta agroecológica é um importante meio de aprendizado e de conhecimento que pode contribuir na conscientização sobre a possibilidade de produzir alimentos de forma saudável e ecologicamente correta, através da interação entre a universidade e a comunidade escolar. Essa estratégia possibilita assim diversas atividades pedagógicas, despertando nos alunos o interesse em práticas alimentares saudáveis e também no cuidado do meio ambiente (FREITAS et al., 2013).

Além disso, trata-se de uma opção para proporcionar às crianças maior proximidade com o solo, plantas e tudo que envolve a produção de alimentos. Pois através do contato com as práticas de produção é possível desenvolver diversas atividades, fazendo com que a teoria e a prática caminhem juntas, de forma que elas aprendam e elevem seus conhecimentos. Com os alunos orientados e com o aprendizado adquirido através da união entre os acadêmicos, professores e os alunos das escolas, as crianças são estimuladas a ter uma alimentação mais saudável e a estarem conscientes de que é possível produzir um alimento nutritivo e saboroso de forma sustentável. Além disso, levar estes conhecimentos até seus lares e assim despertar o interesse em todos os familiares (CRIBB, 2010; MORGADO, SANTOS, 2008; LOMPA, 2016).

Outro fato que reforça ainda mais o incentivo para as crianças aprenderem sobre a produção de alimentos é o Programa "Horta na Escola". O qual foi instituído pelo projeto de lei número 4.516 de 2012 que pede a inclusão do inciso VII no art. 11 da Lei no 9394 de 1996 que 
estabelece as diretrizes e bases da educação nacional. O programa tem como objetivo incentivar a utilização de hortas nas escolas, para que estas possibilitem aos alunos terematividades práticas relacionadas a temas sobre fotossíntese, formação do solo, ciclos biogeoquímicos, interação solo, planta, atmosfera, entre outros, além de garantir um maior desenvolvimento e aprendizado. Desta forma o projeto AgroAção pode servir como estimulo e ajudar as escolas e alunos a terem suas hortas escolares (COMISSÃO DE EDUCAÇÃO, 2012).

Desta forma, o objetivo do projeto AgroAção é promover o desenvolvimento de atividades visando demonstrar estratégias de produção de alimentos de forma sustentável, bem como promover a visitação a trilha ecológica com vistas a despertar o interesse sobre a conservação da biodiversidade.

\section{MATERIAIS E MÉTODOS}

As atividades e visitas do referido projeto foram realizadas na Horta Agroecológica e na Trilha do Pessegueirinho localizadas na Universidade Federal de Santa Catarina, Campus de Curitibanos, tais atividades foram iniciadas em abril de 2016, e vêm sendo mantidas até o momento.

A horta agroecológica possui uma área de $600 \mathrm{~m}^{2}$, sendo utilizada desde 2013 para fins didáticos, de pesquisa e extensão. A horta apresenta-se como experiência prática, uma vez que mantém várias espécies de plantas de interesse alimentar. Outro diferencial da horta é que a mesma abriga PANC’s (Plantas Alimentícias Não Convencionais) como, por exemplo, o caruru, capuchinha, dente de leão, folha gorda, entre outras (Tabela 01). As plantas são cultivadas com base nos preceitos Agroecológicos, buscando a produção de alimentos saudáveis, aproveitando os recursos disponíveis e sem o uso de produtos químicos de alta solubilidade.

As visitas realizadas na horta iniciam em sala de aula (Figura 1A), onde através de recursos audiovisuais promove-se a apresentação do projeto e seus objetivos, além da introdução a abordagem de produção de base agroecológica e consumo consciente. Após esse período, já na horta e com a ajuda dos guias é realizada a apresentação das espécies frutíferas, olerícolas, medicinais e, também ocorre a apresentação de conceitos como, espécies companheiras, compostagem, consórcio de culturas, entre outros.

Já a Trilha do Pessegueirinho foi estabelecida no ano de 2010, possui um trajeto circular de 620 metros, com várias espécies da flora nativa sendo que algumas são identificadas com placas contendo o nome científico, popular e família, justamente para permitir um aprofundamento das discussões sobre a relevância da flora local. Ademais, a trilha também possui placas informativas 
contendo a classificação do solo e suas características. Durante o percurso da trilha é disponibilizado aos visitantes um folder informativo com informações complementares sobre os atrativos da trilha. Nesse sentido, a trilha oferece uma série de temas para aprendizado e descoberta sobre os ecossistemas naturais, bem como sua inter-relação com a produção agropecuária. 
Tabela 01-Lista de espécies vegetais cultivadas na Horta Agroecológica do Campus de Curitibanos/UFSC, juntamente com sua finalidade de uso e curiosidades.

\begin{tabular}{|c|c|c|c|c|}
\hline $\begin{array}{c}\text { Nome } \\
\text { comum }\end{array}$ & Espécie & Família & Uso & Curiosidade \\
\hline Artemísia & Artemisiaabsinthium & Asteraceae & Medicinal e Alimentação & Usada no preparo de aperitivos, distúrbios alimentares, do fígado e vesícula biliar. \\
\hline Arruda & Rutagaveolens & Rutaceae & Medicinal, Ornamental & $\begin{array}{c}\text { Usada na forma de chá para regulação de ciclo menstrual, inflamações na pele, dor de } \\
\text { ouvido e dente, câimbra e doenças no fígado. }\end{array}$ \\
\hline Balsamo & CotyledonorbiculataL. & Crassulaceae & Medicinal e Alimentação & $\begin{array}{l}\text { Tem atividade anti- inflamatórias em casos de queimaduras da pele. Chá reduz azia e } \\
\text { pode ser usado no tratamento de gastrite. }\end{array}$ \\
\hline Batata yacon & Smallanthussonchifoliu & Asteraceae & Medicinal e Alimentação & Reduz a glicose no sangue, sendo indicada para diabéticos. Rica em potássio e vitamina C. \\
\hline Butieiro & $\begin{array}{l}\text { Butiaeriospatha(Mart. } \\
\text { ExDrude) Becc. }\end{array}$ & Arecaceae & $\begin{array}{l}\text { Medicinal, Ornamental e } \\
\text { Alimentação }\end{array}$ & A polpa do seu fruto é usada na produção de óleos, licor e vinhos. \\
\hline Capim limão & Cymbopogoncitrtus & Poaceae & Medicinal e Alimentação & Tem atividade antimicrobiana. Alivia crises de cólicas uterinas e intestinais. \\
\hline Capuchinha & Trompaeolummajus & Tropaeolaceae & $\begin{array}{l}\text { Medicinal, Ornamental e } \\
\text { Alimentação }\end{array}$ & Flores de alto valor nutritivo, usada como fortificante de cabelos, tratamento pulmão. \\
\hline Carqueja & Baccharistrimera & Asteraceae & Medicinal e Alimentação & Amplamente usada para problemas hepáticos. \\
\hline Caruru & AmaranthusviridisL. & Amaranthaceae & Medicinal e Alimentação & Chás de suas folhas são para fins diuréticos e laxativas. \\
\hline Cavalinha & EquisetumgiganteumL. & Equisetaceae & Medicinal e Alimentação & Amplamente utilizada na medicina caseira, como diurético e para o funcionamento dos rins. \\
\hline Dente de leão & Taraxacumofficinale & Asteraceae & Medicinal e Alimentação & Folhas são usadas como salada. Seve para dores reumáticas, diabetes e prisão de ventre. \\
\hline Erva doce & Foeniculumvulgare & Apiaceae & Medicinal & Suas hastes são comestíveis. Funções digestivas. \\
\hline Folha gorda & Portulacaoleraceae & Portulaceae & Medicinal e Alimentação & $\begin{array}{c}\text { Usada para mastites, hemorroidas e vermífuga. Na medicina indígena é usada } \\
\text { principalmente para diabetes. }\end{array}$ \\
\hline Goiaba serrana & Accasellowiana & Myrtaceae & Medicinal e Alimentação & Fruto comestível. Rica em vitaminas vitais e antioxidades. \\
\hline Hortelã & MarrubiumvulgareL. & Lamiaceae & $\begin{array}{l}\text { Medicinal, Ornamental e } \\
\text { Alimentação } \\
\end{array}$ & Seu chá tem fim expectorante, alivia gripes. Serve como tempero. \\
\hline Marcela & Achyroclinesatureiodes & Asteraceae & $\begin{array}{l}\text { Medicinal, Ornamental e } \\
\text { Alimentação }\end{array}$ & Função anti-inflamatória e analgésica. Combate cólicas, dores articulares e musculares. \\
\hline Oraprónobis & Pereskiaaculeata & Cactaceae & Medicinal e Alimentação & $\begin{array}{l}\text { Suas folhas podem ser consumidas como salada. Rica em proteínas e fortalece o } \\
\text { sistema imunológico. }\end{array}$ \\
\hline Physalis & Physalispubescens & Solanaceae & Medicinal e Alimentação & $\begin{array}{l}\text { Empregada no tratamento caseiro do reumatismo crônico, problemas renais, bexiga e } \\
\text { fígado. Seu fruto é comestível. }\end{array}$ \\
\hline Picão & Bidens pilosa L. & Asteraceae & Medicinal e Alimentação & Tem ampla atividade antibactericida. \\
\hline Tansagem & Palntago major & Plantaginaceae & Medicinal e Alimentação & $\begin{array}{c}\text { É antidiarreica. Usada como chá para bronquite crônica e tratamento de ulceras } \\
\text { péptico. }\end{array}$ \\
\hline
\end{tabular}

Fonte: Livro Plantas medicinais no Brasil: nativas e exóticas dos autores Lorenzi e Matos - 2008 


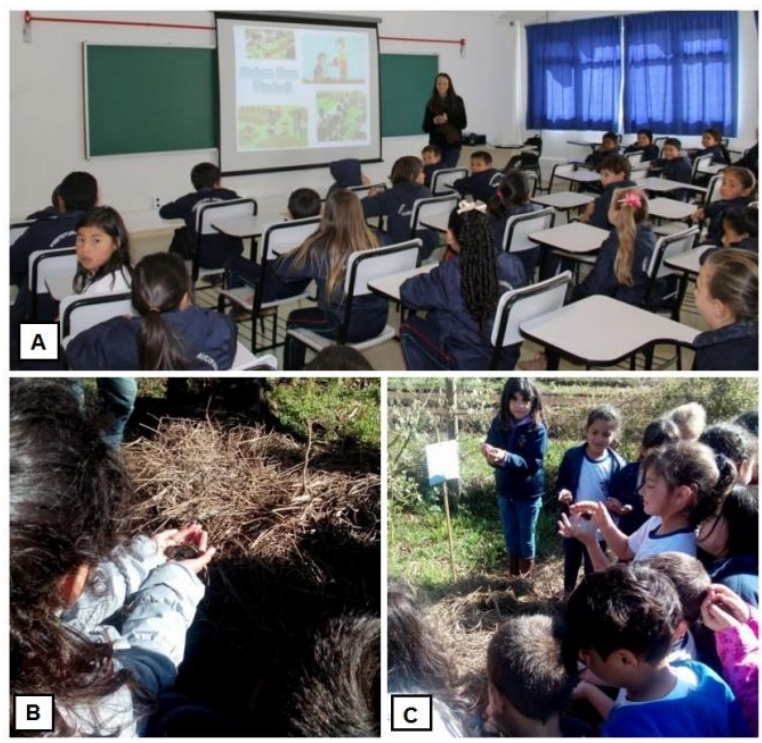

Figura 1 - A) Projeto sendo apresentado às crianças em sala de aula. B) e C) As crianças aprendendo a fazer a compostagem na horta agroecológica do Campus de Curitibanos/UFSC.

Fonte: autores

A visitação na trilha ecológica inicia com apresentação tratando sobre o objetivo da área e os conceitos que envolvem a conservação dos recursos naturais e da biodiversidade. Durante o trajeto existem paradas estratégicas que destacam espécies nativas e suas características; sistemas naturais como o banhado e sua importância; explicam o funcionamento de sistemas de baixo impacto para o manejo da água como o carneiro hidráulico e; também sistemas de proteção de água, a exemplo do sistema caxambu, o qual preserva e protege nascentes de água. Ao final da trilha os alunos visitam o Sistema Agroflorestal, onde é discutido sobre sua função produtiva e ecológica desse sistema de produção.

Nos dias em que as condições climáticas não são favoráveis, as atividades são realizadas em sala de aula, onde as crianças assistem vídeos e praticam atividades lúdicas que tratem sobre os temas alimentação saudável e conservação dos recursos naturais.

\section{RESULTADOS E ANÁLISES}

Os alunos que participaram do projeto (442) apresentaram na sua maioria idade entre 7 a 12 anos. Desde abril de 2016 até o momento, cinco escolas públicas (Tabela 02) de Curitibanos participaram das visitas, tendo um total de 13 visitas com uma média de 34 alunos em cada uma.

Tabela02- Escolas públicas de Curitibanos que participaram do projeto - 2016

\begin{tabular}{|c|c|c|c|}
\hline Data das visitas & Escola & Data das visitas & Escola \\
\hline $29 / 04 / 2016$ & Escola Municipal Getúlio Vargas & $02 / 09 / 2016$ & Núcleo Municipal Rotary \\
\hline
\end{tabular}




\begin{tabular}{|c|c|c|c|}
\hline $06 / 05 / 2016$ & Escola Municipal Getúlio Vargas & $23 / 09 / 2016$ & Núcleo Municipal Rotary \\
\hline $20 / 05 / 2016$ & Escola Municipal Getúlio Vargas & $20 / 10 / 2016$ & $\begin{array}{c}\text { APAE e Escola Estadual Básica } \\
\text { Urbano Salles }\end{array}$ \\
\hline $17 / 06 / 2016$ & $\begin{array}{c}\text { Núcleo Municipal Alírio de } \\
\text { Almeida }\end{array}$ & $21 / 10 / 2016$ & $\begin{array}{c}\text { Colégio Municipal Tereza Lemos } \\
\text { Preto }\end{array}$ \\
\hline $24 / 06 / 2016$ & $\begin{array}{c}\text { Núcleo Municipal Alírio de } \\
\text { Almeida }\end{array}$ & $04 / 11 / 2016$ & Núcleo Municipal Rotary \\
\hline $08 / 07 / 2016$ & $\begin{array}{c}\text { Núcleo Municipal Alírio de } \\
\text { Almeida }\end{array}$ & $18 / 11 / 2016$ & Núcleo Municipal Rotary \\
\hline $26 / 08 / 2016$ & Núcleo Municipal Rotary & & \\
\hline
\end{tabular}

Fonte: elaborado pelos autores

Dentre os resultados obtidos observa-se a relevância de discutir com os alunos sobre uma alimentação saudável. Uma alimentação rica em nutrientes deve começar na infância, para que desde cedo a criança tenha hábitos saudáveis e assim no futuro evite problemas de saúde. Valle e Euclydes (2007) afirmam que a alimentação em grupos, estimula a mudar os hábitos alimentares, assim o incentivo nas escolas é uma ferramenta para que as crianças tenham uma alimentação rica em nutrientes e mais saudável.

Além disso, os alunos observaram a possibilidade de produzir frutas e verduras de qualidade em suas próprias casas. Amaral e Guarim Neto (2008) observaram que as hortas aproximam as pessoas da natureza, mesmo sendo em área urbana, e que as espécies plantadas são importantes fontes de nutrientes, sendo uma alternativa acessível para uma alimentação saudável.

Nas visitas realizadas na horta, os alunos aprendem de forma prática, como fazer a compostagem e onde usá-la (Figura 1 B, C). Foi possível observar que poucos alunos conheciam o processo de compostagem, e a maioria nunca tinha realizado a técnica ou usado o composto. Esses resultados são semelhantes aos observados por Bettencourt et al. (2016) que também observaram através do trabalho de compostagem realizado em escolas públicas, que $67 \%$ dos alunos que participaram não conheciam compostagem.

Além da compostagem, é apresentado as crianças e aos professores como as espécies podem interagir de forma harmônica, garantindo produção através do consórcio de culturas (Figura 2 A e Tabela 1).

Já na Trilha Pessegueirinho (Figura 3 A e B) os alunos aprendem sobre conservação e importância das floretas. Cuba (2007) destaca que a educação ambiental desenvolve a conscientização para que o aluno tenha interesse em conservar e, que isso deve ser construído de uma forma coletiva. Ainda na trilha os alunos aprendem sobre a importância de várias espécies da flora nativa como, por exemplo, a Araucária, Xaxim, Espinheira-santa, Bracatinga, entre outras. Pegoraro e Sorrentino (1998) ressaltam que conservação da flora é sempre uma questão necessária, principalmente devido a aceleração da extinção de espécies e, também das vantagens que a conservação da diversidade de espécies e ecossistemas oferece. 
Outros temas discutidos referem-se às áreas de regeneração natural, importância das áreas úmidas e o dos Sistemas agroflorestais (SAF's) (Figura 3 C e D). Nesses espaços são discutidos temas como a interação entre espécies e a diversidade presente em cada um desses espaços. Mauhs, Marchioretto e Budke (2006) destacam que as áreas úmidas são importantes para a manutenção da diversidade biológica, pois, possuem alta riqueza de espécies tanto da flora quanto da fauna. Higuchiet al. (2006) observaram que áreas de regeneração natural possuem uma ampla diversidade florística no início da regeneração, e que isso é importante para que espécies secundárias se estabeleçam. E o sistema agroflorestal, segundo Abdo, Valeri e Martins (2008), promove como benefício a variabilidade de espécies utilizadas no plantio, o aumento da capacidade do uso da terra, otimização da utilização dos recursos naturais viabilizando a produção, além de trazer benéficos para as características químicas, físicas e biológicas do solo.

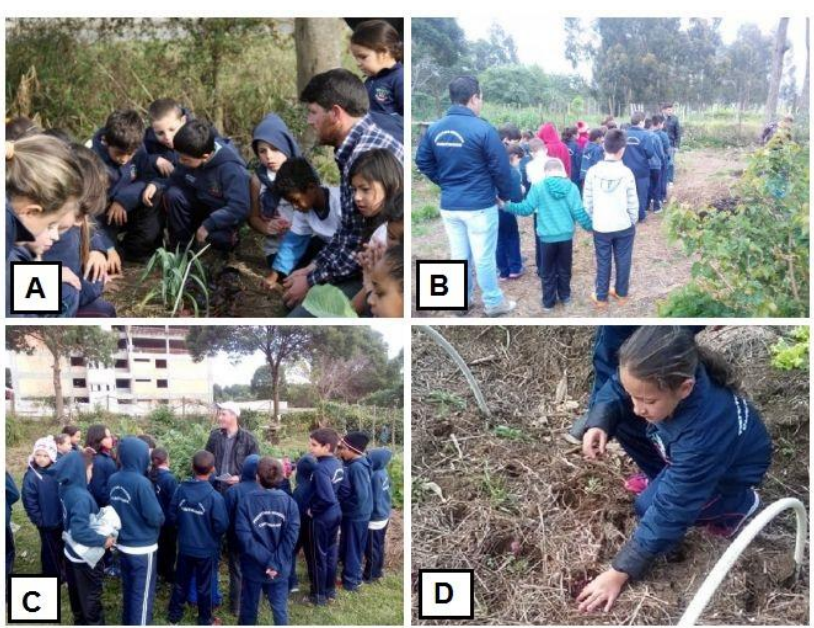

Figura 2 - A) Alunos e professores conhecendo as espécies presentes na horta. B) Apresentação da horta agroecológica para os alunos. C) Apresentação de algumas espécies cultivadas na horta. D) Alunos plantando mudas de beterraba na horta agroecológica do Campus de Curitibanos/UFSC.

Fonte: autores

A horta e a trilha são formas lúdicas para conhecer como as plantas crescem e se desenvolvem, e a importância da conservação das matas, assim acrescentando o aprendizado visto em sala de aula e reforçando a importância da educação ambiental. Morgado e Santos (2008) já observaram que o conhecimento adquirido através das hortas escolares trás uma nova percepção para as crianças de como as plantas crescem e produzem. Adicionalmente, Oliveira (2014) destaca que a educação ambiental é de extrema relevância na formação técnica, mas também cidadã dos jovens do futuro. 


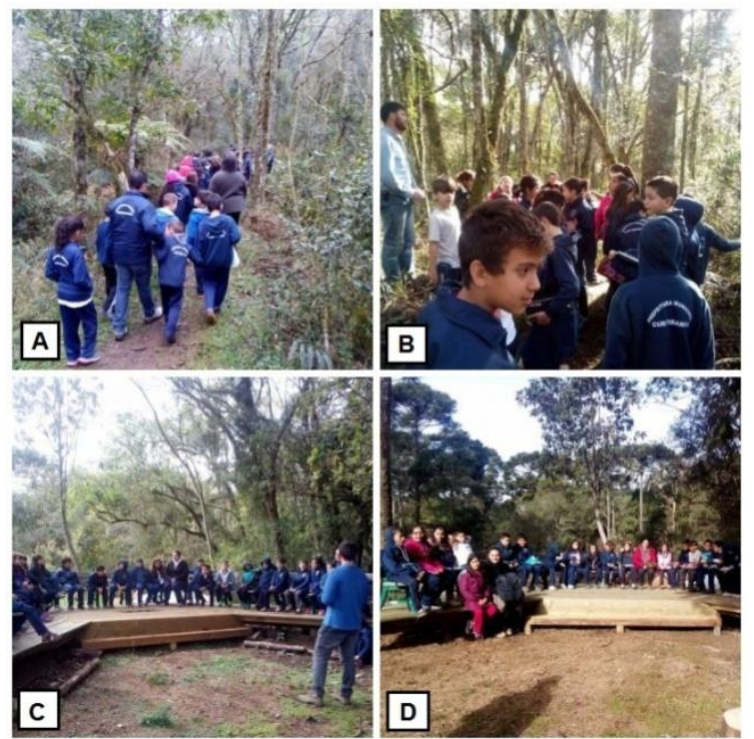

Figura 3 - A) e B) Alunos realizando o percurso da trilha. C) e D) Alunos e professores conhecendo a unidade didática de Sistema Agroflorestal do Campus de Curitibanos/UFSC.

Fonte: autores

\section{CONSIDERAÇÕES FINAIS}

As atividades permitiram a troca de experiência e, para alguns alunos foi o primeiro contato com uma horta ou floresta. Percebe-se através das visitas que as crianças têm pouco contato com a produção de alimentos, desta forma o projeto proporcionou um maior aprendizado e novas experiências para os estudantes. As professoras das turmas demonstraram entusiasmo e interesse pelo projeto, por proporcionar as crianças novas experiências, e almejam que o mesmo continue para que mais crianças tenham a oportunidade de participar das visitas.

Destaca-se também a importância de trazer para o dia a dia dos alunos, assuntos sobre alimentação saudável e conservação da biodiversidade, para que desde crianças tenham um hábito saudável e um maior conhecimento a respeito dos aspectos produtivos, com o propósito de conservar de forma sustentável os recursos naturais disponíveis.

\section{AGRADECIMENTOS}

Prefeitura de Curitibanos-SC e as Escolas: Escola Municipal Getúlio Vargas, Núcleo Municipal Alírio de Almeida, Núcleo Municipal Rotary, APAE Curitibanos, Escola Estadual Básica Urbano Salles e Colégio Municipal Tereza Lemos Preto. A Profa. Dra. Ingrid B.I. de Barros. 


\section{REFERÊNCIAS}

ABDO, Maria Teresa Vilela Nogueira; VALERI, Sérgio Valiengo; MARTINS, Antônio Lúcio Mello. Sistemas agroflorestais e agricultura familiar: uma parceria interessante. Revista Tecnologia \& Inovação Agropecuária, São Paulo, v. 1, n. 2, p.50-59, dez. 2008.

ALTIERI, Miguel; KOOHAFKAN, Parviz. Enduring Farms: Climate Change, Smallholders and Traditional Farming Communities. 6. ed. Malásia: Third World Network, 2008. 63 p. Disponívelem: http://www.twn.my/title/end/pdf/end06.pdf. Acesso em: 03 abr. 2017.

AMARAL, Cleomara Nunes do; GUARIM NETO, Germano. Os quintais como espaços de conservação e cultivo de alimentos: um estudo na cidade de Rosário Oeste (Mato Grosso, Brasil). Boletim do Museu Paraense Emílio Goeldi: Ciências Humanas, Belém, v. 3, n. 3, p.329-341, set. 2008.

BENTO, Isabel Cristina; ESTEVES, Juliana Maria de Melo; FRANÇA, Thaís Elias. Alimentação saudável e dificuldades para torná-la uma realidade: percepções de pais/responsáveis por préescolares de uma creche em Belo Horizonte/MG, Brasil. Ciência \& Saúde Coletiva, Rio de Janeiro, v. 20, n. 8, p.2389-2400, jan. 2015.

BETTENCOURT, Arthur Fernandes et al. Compostagem elaborada a partir de diferentes resíduos nas escolas da rede pública de dompedrito. 2016. Disponível em: http://trabalhos.congrega.urcamp.edu.br/index.php/mpce/article/view/906/929. Acesso em: 14 mar. 2017.

CARVALHO FILHO, José Juliano de. A produção de alimentos e o problema da segurança alimentar. Estudos Avançados, São Paulo, v. 9, n. 24, p.173-193, maio 1995.

COMISSÃO DE EDUCAÇÃO. Congresso. Câmara dos Deputados. Projeto de Lei $\mathbf{n}^{\mathbf{0}} \mathbf{4 . 5 1 6}$, de 2012. Projeto de Lei no 4.516, de 2012. Brasília, DF, 2012. Disponível em: http://www.camara.gov.br/sileg/integras/1083400.pdf. Acesso em: 26 mar. 2017.

CRIBB, Sandra Lucia de Souza Pinto. Contribuições da educação ambiental e horta escolar na promoção de melhorias ao ensino, à saúde e ao ambiente. Rempec: Ensino, Saúde e Ambiente, Niterói, v. 3, n. 1, p.42-60, abr. 2010.

CUBA, Marcos Antonio. Educação ambiental nas escolas. Eccom, Lorena, v. 1, n. 2, p.23-31, jul. 2007.

FREITAS, Helder Ribeiro et al. Horta escolar agroecológica como instrumento de educação ambiental e alimentar na Creche Municipal Dr. Washington Barros - Petrolina/PE. Extramurus: Revista de Extensão da Univasf, Petrolina, v. 1, n. 1, p.155-169, jul. 2013.

HIGUCHI, Pedro et al. Composição florística da regeneração natural de espécies arbóreas ao longo de oito anos em um fragmento de floresta estacional semidecidual, em Viçosa, MG. Revista Árvore, Viçosa, v. 30, n. 6, p.893-904, set. 2006. 
LIMA, Magda Aparecida de. Agropecuária brasileira e as mudanças climáticas globais: caracterização do problema, oportunidades e desafios. Cadernos de Ciência \& Tecnologia, Brasília, v. 19, n. 3, p.451-472, set. 2002.

LOMPA, Marina Arnoldo. O papel das hortas escolares na modificação do comportamento alimentar. 2016. 24 f. TCC (Graduação) - Curso de Nutrição, Universidade Federal do Rio Grande do Sul, Porto Alegre, 2016.

LORENZI, Harri; MATOS, Francisco José de Abreu. Plantas medicinais no Brasil: nativas e exóticas. 2. ed. Nova Odessa: Plantarum, 2008. 869 p.

MARANHÃO, Raquel Veloso de Albuquerque et al. Consumo e motivos para o não consumo de frutas, legumes e verduras por adolescentes e adultos residentes em mesmo domicílio. Nutrire, São Paulo, v. 39, n. 2, p.203-213, ago. 2014.

MAUHS, Julian; MARCHIORETTO, Maria Salete; BUDKE, Jean Carlos.Riqueza e biomassa de macrófitas aquáticas em uma área úmida na planície costeira do Rio Grande do Sul, Brasil. Pesquisas Botânicas, São Leopoldo, v. 57, n. 57, p.289-302, jan. 2006.

MORGADO, Fernanda da Silva; SANTOS, Mônica Aparecida Aguiar dos. A horta escolar na educação ambiental e alimentar: experiência do Projeto Horta Viva nas escolas municipais de Florianópolis. ExtensioUfsc, Florianópolis, v. 5, n. 6, p.1-10, jun. 2008.

OLIVEIRA, Luciana Cristina Montonede. Horta escolar: a realidade das escolas de ArarasSP. 2014. 54 f. Monografia (Especialização) - Curso de Especialização em Ensino de Ciências, Universidade Tecnológica Federal do Paraná, Medianeira, 2014.

PEGORARO, João Luiz; SORRENTINO, Marcos. Programas educativos com flora e fauna (expressões da biodiversidade) e a educação ambiental. ScientiaForestalis, São Paulo, v. 54, n. 54, p.131-142, dez. 1998.

PINTO, Hilton Silveira; ASSAD, Eduardo Delgado. Aquecimento global e cenários futuros da agricultura brasileira. 2009. Disponível em: http://www.greenpeace.org/brasil/pagefiles/3580/eduardo assadi embrapa.pdf. Acesso em: 03 abr. 2017.

VALLE, Janaína Mello Nasser; EUCLYDES, Marilene Pinheiro. A formação dos hábitos alimentares na infância: uma revisão de alguns aspectos abordados na literatura nos últimos dez anos. Revista Aps, Juiz de Fora, v. 10, n. 1, p.1-20, jan. 2007.

WHO; FAO.T Consultation on Diet, Nutrition and the Prevention of Chronic Diseases. Who Library, Geneva, v. 1, n. 1, p.101-101, fev. 2003.

Recebido em: 05/07/2017

Aceito em: 28/06/2018 